\title{
Die starke rechtspolitische Stimme des djb
}

Prof. Dr. Dres. h.c. Hans-Jürgen Papier, Präsident des Bundesverfassungsgerichts, Karlsruhe

Sehr geehrte Frau Präsidentin Wagner,

liebe Teilnehmerinnen des Kongresses des Deutschen Juristinnenbundes,

verehrte Ehrengäste,

ich begrüße Sie auf das Herzlichste im Bundesverfassungsgericht und freue mich, dass der 38. Kongress des Deutschen Juristinnenbundes mit dem vielschichtigen und anspruchsvollen Thema „Integration durch Recht - Frauen mit Migrationshintergrund zwischen Teilhabe und Ausgrenzung “ hier im Sitzungssaal des Bundesverfassungsgerichts eröffnet wird. Ist doch dieser Sitzungssaal in den letzten 40 Jahren geradezu zu einem Symbol des deutschen Rechtsstaates nach 1945 geworden.

Ich freue mich vor allem darüber, so viele Juristinnen aus allen juristischen Berufssparten im Bundesverfassungsgericht versammelt zu sehen. Wenn ich mich in dieser Runde umschaue, so macht das Mut und es liegt geradezu auf der Hand, dass es dem Bundestag und dem Bundesrat in absehbarer Zeit möglich sein wird, die Anzahl der weiblichen Richter des Bundesverfassungsgerichts deutlich zu erhöhen. Hier sollte der Deutsche Juristinnenbund, der ja rechtspolitisch eine starke Stimme hat, etwa im Rahmen von Gesetzgebungsvorhaben, aber auch in verfassungsgerichtlichen Verfahren, diese Stimme auch verstärkt bei den Findungsgesprächen für neue Verfassungsrichterinnen und selbstverständlich auch für neue Verfassungsrichter erheben.

Nachdem ich eben schon die Stellungnahmen des Deutschen Juristinnenbundes und insbesondere seiner Expertinnen in Verfahren vor dem Bundesverfassungsgericht angesprochen habe, ist es mir ein Anliegen, diesen Sachverständigen und auch dem Deutschen Juristinnenbund selbst für dieses Enga-

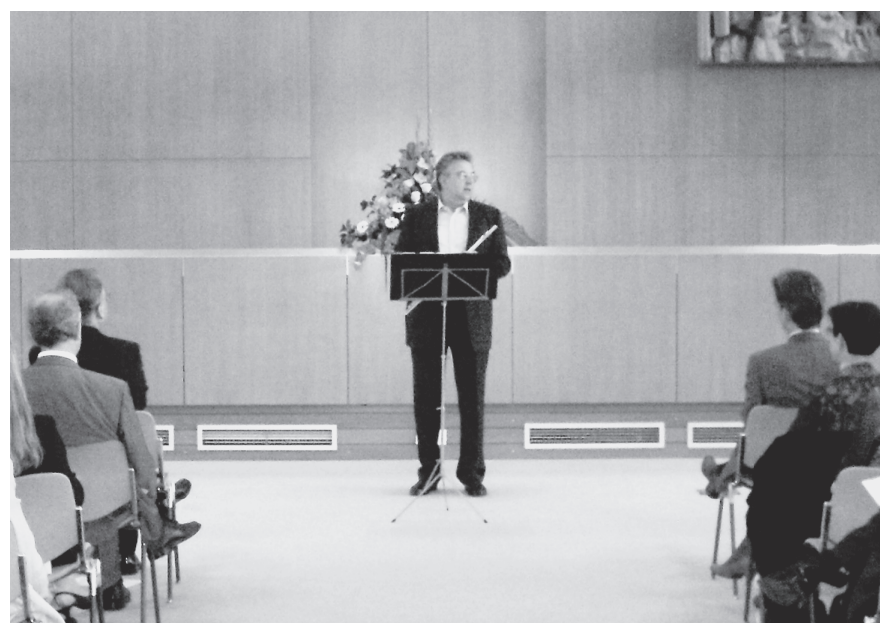

$\Delta$ Eröffnungsveranstaltung des 38. djb-Kongresses im Bundesverfassungsgericht mit Jos Rinck „Der Klang von Räumen“ (Foto: CD). gement zu danken. Ihren verfassungsrechtlichen Sachverstand, aber auch Ihre Erfahrungen im Alltag der Rechtspraxis, etwa bei der juristischen Beratung oder der Tätigkeit in den Fachgerichtsbarkeiten, die in diese Stellungnahmen einfließen, sind für das Bundesverfassungsgericht unverzichtbar und stellen regelmäßig wichtige Erkenntnisquellen dar. Dabei wissen wir, dass diese sachverständigen Stellungnahmen häufig neben der oftmals großen Arbeitsbelastung im Hauptamt erstellt werden. Diese Äußerungen und Stellungnahmen sind ein Zeichen für das große juristische, rechts- und gesellschaftspolitische Engagement, das für unser Gemeinwesen unverzichtbar ist. Gern greife ich daher die sich mir durch dieses Grußwort eröffnete Gelegenheit auf, um dem Deutschen Juristinnenbund und vor allem all jenen Mitgliedern, die die Aufgabe des Erarbeitens von Stellungnahmen auf sich genommen haben, zu danken. Ihre fundierten, zum Teil auch exponierten Stellungnahmen sind immer eine Bereicherung in den Beratungen der Senate. Sie haben so die Rechtsprechung des Bundesverfassungsgerichts mit beeinflusst und mitgeprägt und bisweilen wohl auch einzelne Senatsmitglieder, vielleicht auch den ganzen Senat geradezu vorangetrieben, über neue Wege nachzudenken.

Aber nicht nur auf diese Weise hat der Deutsche Juristinnenbund im Bundesverfassungsgericht gewirkt. Vielmehr geschieht dieses auch dadurch, dass zahlreiche Richterinnen des Bundesverfassungsgerichts zugleich Mitglieder des Deutschen Juristinnenbundes waren und auch - wenn ich es richtig sehe - sind. Drei dieser Kolleginnen, die hier bahnbrechend und wegweisend gewirkt haben, möchte ich bei diesem besonderen Anlass erwähnen und würdigen. Alle Anwesenden, vor allem die drei anwesenden aktiven Richterinnen des Bundesverfassungsgerichts, aber auch die inzwischen in Straßburg tätige Kollegin Jaeger bitte ich um Verständnis, dass meine Wahl auf die ehemaligen Richterinnen Erna Scheffler, Wiltraut Ruppvon Brünneck und Helga Seibert gefallen ist. Frau Jaegers Wirken im Bundesverfassungsgericht habe ich bei ihrem Ausscheiden an dieser Stelle gewürdigt und ihre jetzige Tätigkeit am Europäischen Gerichtshof für Menschenrechte verbietet es, trotz des kooperativen Verhältnisses zwischen beiden Gerichten, mich zu ihrer Arbeit öffentlich zu äußern, obgleich hierzu viel Lobendes zu sagen wäre. Die Kolleginnen Osterloh, Hohmann-Dennhardt und Lübbe-Wolff wissen um die Usance, dass an diesem Ort und dieser Stelle die Arbeit eines Mitglieds des Gerichts erst nach seinem Ausscheiden gewürdigt wird - frühestens, dann selbstverständlich noch einmal beim Ableben.

Lassen Sie mich nun zu der ersten Richterin des Bundesverfassungsgerichts, Frau Erna Scheffler, kommen. Schon vor ihrer Ernennung zur Richterin des Bundesverfassungsgerichts machte Erna Scheffler durch ihr Referat auf dem 38. (!) Deutschen Juristentag 1950 keinen Hehl aus ihrer Grundhaltung; sie machte schon damals von sich reden. Die zutage tretende 


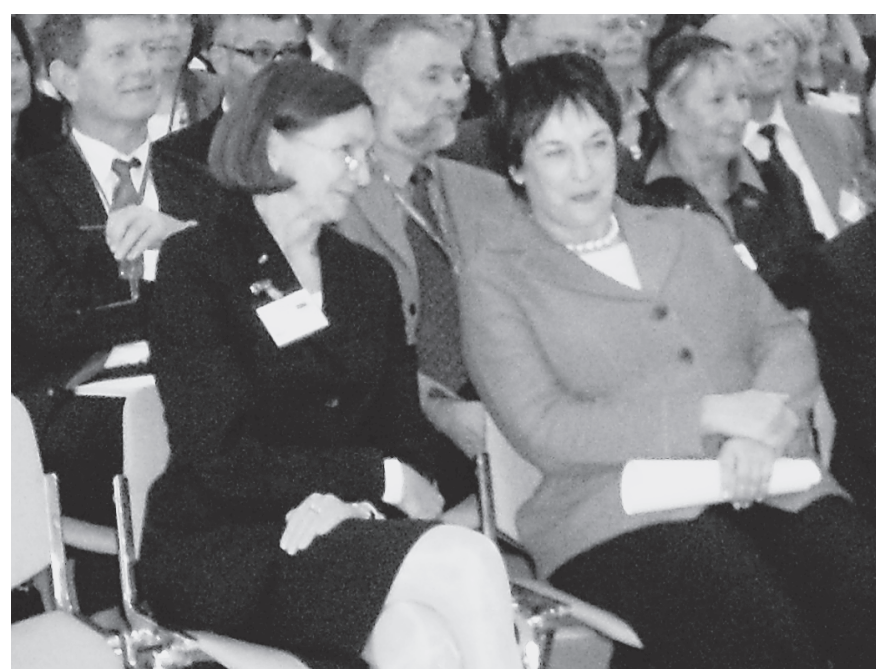

$\Delta$ Präsidentin Jutta Wagner (links) im Gespräch mit Bundesjustizministerin Brigitte Zypries (Foto: CD)

Überzeugungskraft und ihr Engagement für die Gleichberechtigung in allen Rechtsgebieten waren wohl auch die Basis dafür, dass das Bundesverfassungsgericht die Gleichberechtigung von Mann und Frau in zahlreichen Entscheidungen in Verfassungswirklichkeit umgestaltet hat. Als Berichterstatterin hat Erna Scheffler folgende wichtige Entscheidungen vorbereitet:

- Ausspruch, dass ab 1. April 1953 Mann und Frau auch im Bereich von Ehe und Familie gleichberechtigt sind und das entgegenstehende bürgerliche Recht außer Kraft getreten war $^{1}$,

- Verfassungswidrigkeit der früheren Regelungen zur Zusammenveranlagung im Steuerrecht ${ }^{2}$,

- Verfassungswidrigkeit der Höfeordnung, soweit diese eine Bevorzugung des männlichen Erben vorsah ${ }^{3}$,

- Unvereinbarkeit von Regelungen der Sozialversicherung, soweit diese den Wert der Leistungen als Mutter, Hausfrau und Mithelfende in der Sozialversicherung unberücksichtigt lassen ${ }^{4}$.

Besonders hervorzuheben ist aber die Entscheidung zum Stichentscheid des Vaters, die Erna Scheffler in Vertretung des erkrankten Präsidenten im Juli 1959 selbst verkündete (BVerfGE 10, 59). Um den juristischen und sozialen Kontext und damit zugleich die - trotz der Geltung des Artikel 3 Abs. 2 GG - revolutionäre Dimension dieser Entscheidung wie auch vorangegangener Entscheidungen des Bundesverfassungsgerichts erkennbar zu machen, ist es aufschlussreich, sich das noch in den 1950er Jahren bestehende Diskussionsklima in Sachen Gleichberechtigung von Mann und Frau in Erinnerung zu rufen. Bereits im Dezember 1953 hatte das Bundesverfassungsgericht festgestellt, dass Mann und Frau auch ohne Ausführungsgesetz seit dem Ablauf der im Grundgesetz gesetzten Frist (31. März 1953) aufgrund von Art. 3 Abs. 2 GG gleichberechtigt sind. ${ }^{5}$ Der BGH hatte sich in seiner damaligen
Stellungnahme ebenfalls für dieses Ergebnis ausgesprochen 6 und dabei den seinerzeitigen juristischen Streitstand in Deutschland zusammengefasst. Dort ist von Auffassungen zum Verhältnis von Mann und Frau und zur Rolle des Mannes als „Haupt der Familie“ die Rede, die mit folgenden Worten näher umrissen werden:

„Es wäre in hohem Maße gefährlich, die ehemännliche Familienleitung aufzuheben und, was die Entscheidung der gemeinsamen ehelichen Angelegenheiten angeht, die Anarchie in die Ehen einzuführen. Das würde bedeuten, dass auf diesem ungemein bedeutsamen Gebiete nicht das Recht, sondern die Willkür und die Gewalt herrschen würden; ein schlechthin unvollziehbarer Gedanke7.“

Dieser Bericht des BGH über eine in den 1950er Jahren vertretene Ansicht macht deutlich, dass damals Verfassungsrecht und Verfassungswirklichkeit auch in den Köpfen so mancher Juristen noch weit auseinanderklafften. Dass heute gerade im Hinblick auf Artikel 3 Abs. 2 GG Verfassungsrecht und Verfassungswirklichkeit in vielen Bereichen nahezu deckungsgleich sind, verdanken wir auch Richterinnen wie Wiltraut Rupp-von Brünneck und Helga Seibert.

Frau Rupp-von Brünneck war ebenso wie Frau Seibert während ihrer gesamten Amtszeit einzige Richterin des Bundesverfassungsgerichts. Diese Rolle gehört heute und hoffentlich auch zukünftig der Vergangenheit an, obgleich zu bemerken ist, dass Frau Kollegin Hohmann-Dennhardt zurzeit die einzige Richterin im Ersten Senat ist. Auch diese Rolle dürfte bisweilen nicht immer leicht zu tragen sein; ich verrate allerdings kein Geheimnis, wenn ich sage, dass Frau HohmannDennhardt damit bestens fertig wird. Allen drei genannten Richterinnen war bzw. ist die Berichterstattung für alle Verfahren aus dem Familienrecht anvertraut. Wiltraut Rupp-von Brünneck hat als Berichterstatterin auf diesem Gebiet die wegweisenden Entscheidungen zum Recht des nichtehelichen Kindes $^{8}$ und zur Reform des Scheidungsrechts ${ }^{9}$ vorbereitet. Ruppvon Brünnecks Sondervoten zu Entscheidungen aus anderen Gebieten sind heute noch wegweisend und wurden in einem Buch zu ihren Ehren gesondert herausgegeben. Noch immer aktuell sind zum Beispiel ihre Sondervoten zum Abhörurteil ${ }^{10}$ und zur Mephisto-Entscheidung ${ }^{11}$, das eine Renaissance in dem Sondervotum zur Esra-Entscheidung erfahren hat, sowie das Sondervotum zum Schwangerschaftsabbruch ${ }^{12}$.

Abschließend möchte ich auch die Verfassungsrichterin Helga Seibert würdigen. Auch sie war Berichterstatterin für

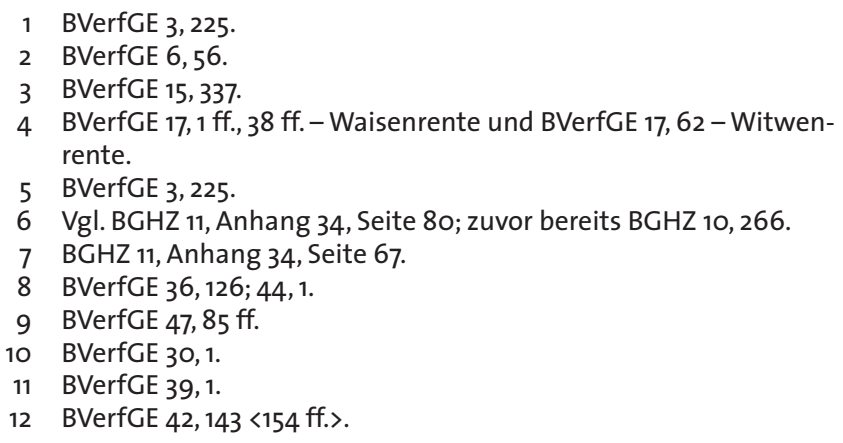


das Gebiet des Familienrechts und hat hier gleichfalls wichtige und wegweisende Entscheidungen vorbereitet. Erwähnenswert sind insbesondere der Beschluss des Ersten Senats zum Sorgerechtsverlust der Mutter bei Ehelichkeitserklärung des Kindes ${ }^{13}$ sowie der Beschluss zur Bestimmung des Familiennamens bei Eheschließungen ${ }^{14}$. Beide Beschlüsse waren Anstoß für sehr grundlegende Änderungen des Familien- und $\mathrm{Na}$ mensrechts und veranlassten den Gesetzgeber, wenn auch zum Teil nur sukzessive und nur nach erneuter Entscheidung des Bundesverfassungsgerichts, das Recht maßgeblich zu ändern. Der Beschluss zum Sorgerechtsverlust der Mutter bei Ehelichkeitserklärung des Kindes war der entscheidende Anstoß für die Erarbeitung eines Gesetzentwurfs, der auch nicht verheirateten Eltern generell die Möglichkeit einer gemeinsamen Sorge für die Kinder einräumt. Beim Namensrecht führte die von Helga Seibert vorbereitete Entscheidung zunächst einmal dazu, dass die Frau das Recht erhielt, ihren Mädchennamen beizubehalten. Es bedurfte dann über Jahre versetzt weiterer - letztlich vom Bundesverfassungsgericht erzwungener - Ge- setzesänderungen bis zum heutigen Recht, das jedem Ehepartner die Beibehaltung seines Namens ermöglicht.

Diese drei großen Richterpersönlichkeiten, die bekanntermaßen von Statur her zum Teil gar nicht groß, sondern zierlich und klein waren, sind für uns hier im Bundesverfassungsgericht heute - wie auch viele andere - immer noch Vorbild und Ansporn. Ich bin mir sicher, dass es Vielen hier im Raume ebenso geht.

In diesem Sinne möchte ich Sie anspornen und ermutigen, Ihren rechtspolitischen Erfolgskurs fortzusetzen; ich wünsche Ihnen zunächst einmal interessante und spannende Referate, offene und kontroverse Diskussionen und vor allem neue Erkenntnisse, die möglicherweise auch einmal die Rechtsprechung des Bundesverfassungsgerichts beeinflussen werden. Ich wünsche Ihnen ein gutes Gelingen des 38. Kongresses des Deutschen Juristinnenbundes.

\footnotetext{
13 BVerfGE 84, 168.
}

14 BVerfGE 84, 9 ff.

\section{Wir müssen auch in Zukunft eine Menge tun}

\section{Brigitte Zypries MdB}

Bundesministerin der Justiz, Berlin/Darmstadt

Sehr geehrter Herr Präsident des Bundsverfassungsgerichts, sehr geehrte Damen und Herren, liebe Jutta Wagner,

ich bin gerne zu Ihnen gekommen, denn Sie haben sich ein besonders wichtiges Thema vorgenommen: Die bessere Integration von Migrantinnen ist eine enorme Herausforderung für unsere Gesellschaft und ich bin sehr dankbar, dass sich der Juristinnenbund dieser Aufgabe mit soviel Engagement annimmt. Wie brisant die "Integration durch Recht" ist, liegt auf der Hand. Ich erinnere nur an die Reform des Staatsangehörigkeitsrechts, an das Zuwanderungsgesetz und an die politischen Kampagnen, die es gegen diese Projekte gegeben hat. Für Rot-Grün ging es damals darum, gesellschaftliche Realität und Recht wieder stärker miteinander in Einklang zu bringen. Mit den Reformgesetzen haben wir erstmals anerkannt, dass Deutschland ein Einwanderungsland ist. Und wir haben deutlich gemacht, dass wir etwas dafür tun müssen, um diejenigen, die dauerhaft bei uns bleiben, besser zu integrieren. Auch die große Koalition hat sich mit dem Thema Integration befasst, aber um ganz ehrlich zu sein: Herausgekommen im Sinne des Rechts ist dabei nicht viel. Wenn die Bundeskanzlerin zu einem Integrationsgipfel einlädt, aber ihr Innenminister festlegt, dass dort nicht über das Aufenthaltsrecht gesprochen wird, dann ist klar, dass es eher um weiche Themen geht. Wichtig war aber auch, dass die Teilnehmer eine Vorbild- funktion für den Dialog über eine gelungene Integration gegeben haben.

Ich meine aber, die Bundespolitik ist dafür verantwortlich, die gesetzlichen Rahmenbedingungen so zu setzen, dass Integration auch wirklich gelingen kann. Und dafür müssen wir auch in Zukunft eine Menge tun. Ich meine, wir sollten zum Beispiel im Staatsbürgerschaftsrecht die sogenannte Optionslösung abschaffen. Junge Migrantinnen und Migranten sollen nicht länger gezwungen werden, sich zwischen dem deutschen Pass und der Staatsangehörigkeit ihrer Eltern zu entscheiden. Wer nur Deutscher auf Zeit ist, wer mit spätestens 23 Jahren seinen deutschen Pass vielleicht wieder verliert, der wird sich hier nicht wirklich heimisch fühlen. Wir sollten deshalb bereit sein, doppelte Staatsangehörigkeiten häufiger hinzunehmen und das Optionsmodell abschaffen. Auch am Aufenthaltsrecht müssen wir weiter arbeiten - vor allem im Interesse der Frauen. Es darf nicht sein, dass Frauen, die Deutschland verlassen, schon nach sechs Monaten ihren Aufenthaltsstatus verlieren. Wir alle wissen von den Fällen: Junge Frauen, die in Deutschland aufgewachsen sind, werden im Heimatland ihrer Eltern zwangsweise verheiratet. Wenn diese Frauen aus solch einer Ehe ausbrechen und zurück nach Deutschland wollen, dann dürfen unsere Gesetze das nicht verhindern. Seinen Lebenspartner selbstbestimmt zu wählen, das ist nämlich auch ein Menschenrecht. Ich meine deshalb: Für diese Fälle der Heiratsverschleppung brauchen wir eine Sonderregelung im Aufenthaltsrecht. 